Research Article

\title{
Fixed Point Theorems for Generalized $\theta-\phi-$ Expansive Mapping in Rectangular Metric Spaces
}

\author{
Mohamed Rossafi $\left(\mathbb{D},{ }^{1}\right.$ Abdelkarim Kari $\mathbb{D}^{2}$ El Miloudi Marhrani $\mathbb{D}{ }^{2}$ \\ and Mohamed Aamri ${ }^{2}{ }^{2}$ \\ ${ }^{1}$ LASMA Laboratory Department of Mathematics Faculty of Sciences, Dhar El Mahraz University Sidi Mohamed Ben Abdellah, \\ Fes, Morocco \\ ${ }^{2}$ Laboratory of Algebra, Analysis and Applications Faculty of Sciences Ben M'Sik, Hassan II University, Casablanca, Morocco
}

Correspondence should be addressed to Abdelkarim Kari; abdkrimkariprofes@gmail.com

Received 6 December 2020; Accepted 3 March 2021; Published 12 March 2021

Academic Editor: Gueo Grantcharov

Copyright (c) 2021 Mohamed Rossafi et al. This is an open access article distributed under the Creative Commons Attribution License, which permits unrestricted use, distribution, and reproduction in any medium, provided the original work is properly cited.

In this paper, we present the notion of $\theta-\phi$ - expansive mapping in complete rectangular metric spaces and study various fixed point theorems for such mappings. The presented theorems extend, generalize, and improve many existing results in the literature.

\section{Introduction}

The problem of fixed points of mapping with an adequate contractive condition has been the focal point of a rigorous research activity. It has an extensive applications in different areas such as nonlinear and adaptive control systems, parametrized estimation problems, fractal image decoding, and convergence of recurrent networks.

In 1922, the Banach contraction principle [1] came into existence, which is a very famous theorem in nonlinear analysis and has many useful applications.

Several mathematicians generalized the Banach contraction principle in two major directions, one by stating the conditions on the mapping $T$ and second allowing the set $X$ to have a more general structure [2-7].

In 1984, Wang et al. [8] presented some interesting work on expansion mappings in metric spaces. Recently, Kumar et al. [9] introduced a new notion of generalized $(\alpha, \psi)$ - expansive mappings and established some fixed point theorems for such mappings in complete generalized metric spaces.

In this paper, inspired by the idea of $\theta-\phi$-contraction introduced by Zheng et al. [10] in metric spaces, we presented $\theta-\phi-$ expansive mapping and establish various fixed point theorems for such mappings in complete rectangular metric spaces. Our theorems extend, generalize, and improve many existing results.

\section{Preliminaries}

In this section, we recall some definitions and results that will be used to prove our main results.

By an expansion mapping [8] on a metric space $(X, d)$, we understand a mapping $T: X \longrightarrow X$ satisfying for all $x, y \in X$ :

$$
d(T x, T y) \geq k d(x, y)
$$

where $k$ is a real in $] 1,+\infty[$.

In 2000 Branciari [2] introduced the concept of rectangular metric spaces as follows:

Definition 1 (see [11]). Let $X$ be a nonempty set and $d: X$ $\times X \longrightarrow \mathbb{R}^{+}$be a mapping such that for all $x, y \in X$ and for all distinct points $u, v \in X$, each of them different from $x$ and $y$, one has

(i) $d(x, y)=0$ if and only if $x=y$

$$
d(x, y)=d(y, x)
$$

(ii) $d(x, y) \leq d(x, u)+d(u, v)+d(v, y)$ (the rectangular inequality) 
Then, $(X, d)$ is called a rectangular metric space.

Definition 2 (see [12]). Let $T: X \longrightarrow X$ and $\alpha, \eta: X \times X$ $\longrightarrow[0,+\infty[$. We say that $T$ is a triangular $(\alpha, \eta)$-admissible mapping if

$\left(\mathrm{T}_{1}\right) \alpha(x, y) \geq 1 \Longrightarrow \alpha(T x, T y) \geq 1, x, y \in X$

$\left(\mathrm{T}_{2}\right) \eta(x, y) \leq 1 \Longrightarrow \eta(T x, T y) \leq 1, x, y \in X$

$\left(\mathrm{T}_{3}\right)\left\{\begin{array}{l}\alpha(x, y) \geq 1 \\ \alpha(y, z) \geq 1\end{array} \Longrightarrow \alpha(x, z) \geq 1\right.$ for all $x, y, z \in X$

$\left(\mathrm{T}_{4}\right)\left\{\begin{array}{l}\eta(x, y) \leq 1 \\ \eta(y, z) \leq 1\end{array} \Longrightarrow \eta(x, z) \leq 1\right.$ for all $x, y, z \in X$

Definition 3 (see [12]). Let $(X, d)$ be a rectangular metric space and let $\alpha, \eta: X \times X \longrightarrow[0,+\infty[$ be two mappings.

(a) $T$ is $\alpha$-continuous mapping on $(X, d)$, if for given point $x \in X$ and sequence $\left\{x_{n}\right\}$ in $X, x_{n} \longrightarrow x$ and $\alpha$ $\left(x_{n}, x_{n+1}\right) \geq 1$ for all $n \in \mathbb{N}$ imply that $T x_{n} \longrightarrow T x$

(b) $\mathrm{T}$ is $\eta$ subcontinuous mapping on $(X, d)$, if for given point $x \in X$ and sequence $\left\{x_{n}\right\}$ in $X, x_{n}$ $\longrightarrow x$ and $\eta\left(x_{n}, x_{n+1}\right) \leq 1$ for all $n \in \mathbb{N}$ imply that $\mathrm{T} x_{n} \longrightarrow T x$

(c) $\mathrm{T}$ is $(\alpha, \eta)$-continuous mapping on $(X, d)$, if for given point $x \in X$ and sequence $\left\{x_{n}\right\}$ in $X, x_{n} \longrightarrow x$ and $\alpha\left(x_{n}, x_{n+1}\right) \geq 1$ or $\eta\left(x_{n}, x_{n+1}\right) \leq 1$ for all $n \in \mathbb{N}$ imply that $T x_{n} \longrightarrow T x$

Recently, Hussain et al. [13] gave the following definitions.

Definition 4 (see [13]). Let $d(X, d)$ be a rectangular metric space and let $\alpha, \eta: X \times X \longrightarrow[0,+\infty[$ be two mappings. The space $X$ is said to be

(a) $\alpha$-complete, if every Cauchy sequence $\left\{x_{n}\right\}$ in $X$ with $\alpha\left(x_{n}, x_{n+1}\right) \geq 1$ for all $n \in \mathbb{N}$ converges in $X$

(b) $\eta$ - sup - complete, if every Cauchy sequence $\left\{x_{n}\right\}$ in $X$ with $\eta\left(x_{n}, x_{n+1}\right) \leq 1$ for all $n \in \mathbb{N}$ converges in $X$

(c) $(\alpha, \eta)$-complete, if every Cauchy sequence $\left\{x_{n}\right\}$ in $X$ with $\alpha\left(x_{n}, x_{n+1}\right) \geq 1$ or $\eta\left(x_{n}, x_{n+1}\right) \leq 1$ for all $n \in$ $\mathbb{N}$ converges in $X$

Definition 5 (see [13]). Let $(X, d)$ be a rectangular metric space and let $\alpha, \eta: X \times X \longrightarrow[0,+\infty[$ be two mappings. The space $(X, d)$ is said to be

(a) $(X, d)$ is $\alpha$-regular, if $x_{n} \longrightarrow x$, where $\alpha\left(x_{n}, x_{n+1}\right) \geq 1$ for all $n \in \mathbb{N}$ implies $\alpha\left(x_{n}, x\right) \geq 1$ for all $n \in \mathbb{N}$

(b) $(X, d)$ is $\eta$ - subregular, if $x_{n} \longrightarrow x$, where $\eta\left(x_{n}, x_{n+1}\right)$ $\leq 1$ for all $n \in \mathbb{N}$ implies $\eta\left(x_{n}, x\right) \leq 1$ for all $n \in \mathbb{N}$

(c) $(X, d)$ is $(\alpha, \eta)$-regular, if $x_{n} \longrightarrow x$, where $\alpha\left(x_{n}, x_{n+1}\right)$ $\geq 1$ or $\eta\left(x_{n}, x_{n+1}\right) \leq 1$ for all $n \in \mathbb{N}$ implies that $\alpha$ $\left(x_{n}, x\right) \geq 1$ or $\eta\left(x_{n}, x\right) \leq 1$ for all $n \in \mathbb{N}$
The following definitions were given by Jleli et al. in [14].

Definition 6 (see [14]). Let $\Theta$ be the family of all functions $\theta:] 0,+\infty[\longrightarrow] 1,+\infty[$ such that

$\left(\theta_{1}\right) \theta$ is increasing, i.e., for all $x, y \in \mathbb{R}^{+}$such that $x<y$, $\theta(x)<\theta(y)$

$\left(\theta_{2}\right)$ For each sequence $\left.x_{n} \in\right] 0,+\infty[$

$$
\lim _{n \rightarrow 0} x_{n}=0 \text {, if and only if } \lim _{n \rightarrow \infty} \theta\left(x_{n}\right)=1
$$

$\left(\theta_{3}\right) \theta$ is continuous

Definition 7 (see [14]). Let $(X, d)$ be a rectangular metric space and $T: X \longrightarrow X$ be a mapping.

$T$ is said to be a $\theta$-contraction if there exist $\theta \in \Theta$ and $k \in] 0,1[$ such that for any $x, y \in X$,

$$
d(T x, T y)>0 \Longrightarrow \theta[d(T x, T y)] \leq[\theta(d(x, y))]^{k} .
$$

Recently, Zheng et al. [10] considered the following family of functions and introduced the notion of $\theta-\phi-$ contraction of metric spaces:

Definition 8 (see [10]). Let $\Phi$ be the family of all functions $\phi:[1,+\infty[\longrightarrow[1,+\infty[$, such that

$\left(\theta_{1}\right) \phi$ is increasing

$\left(\theta_{2}\right)$ For each $\left.t \in\right] 1,+\infty\left[, \lim _{n \rightarrow \infty} \phi^{n}(t)=1\right.$;

$\left(\theta_{3}\right) \phi$ is continuous.

Lemma 9 (see [10]). If $\phi \in \Phi$. Then, $\phi(1)=1$ and $\phi(t)<t$ for all $t>1$.

Definition 10 (see [10]). Let $(X, d)$ be a metric space and $T: X \longrightarrow X$ be a mapping.

$T$ is said to be a $\theta-\phi$-contraction if there exist $\theta \in \Theta$ and $\phi \in \Phi$ such that for any $x, y \in X$,

$$
d(T x, T y)>0 \Longrightarrow \theta[d(T x, T y)] \leq \phi(\theta[N(x, y)]),
$$

where

$$
N(x, y)=\max \{d(x, y), d(x, T x), d(y, T y)\} .
$$

Now, we recall the definition of generalized $(\alpha, \psi)-$ expansive mapping.

Let $\Psi$ be the family of functions $\psi:[0, \infty) \longrightarrow[0, \infty)$ which satisfy the following:

(i) $\psi$ is upper semicontinuous

(ii) $\left(\psi^{n}(t)\right)_{n \in \mathbb{N}}$ converges to 0 as $n \longrightarrow \infty$, for all $t>0$

(iii) $\psi(t)<t$, for any $t>0$

Definition 11 (see [10]). Let $(X, d)$ be a generalized metric space and $T: X \longrightarrow X$ be a given mapping. $T$ is said to be a generalized $(\alpha, \psi)$-expansive mapping of type I if there 
exists two functions $\alpha: X \times X \longrightarrow[0, \infty[$ and $\psi \in \Psi$ such that for all $x, y \in X$,

$$
\psi(d(T x, T y)) \geq \alpha(x, y) M(x, y),
$$

where

$$
M(x, y)=\max \{d(x, y), d(x, T x), d(y, T y)\} .
$$

Definition 12 (see [10]). Let $(X, d)$ be a generalized metric space and $T: X \longrightarrow X$ be a given mapping. $T$ is said to be a generalized $(\alpha, \psi)$ - expansive mapping of type II if there exists two functions $\alpha: X \times X \longrightarrow[0, \infty[$ and $\psi \in \Psi$ such that for all $x, y \in X$,

$$
\psi(d(T x, T y)) \geq \alpha(x, y) N(x, y),
$$

where

$$
N(\mathrm{x}, y)=\min \left\{d(x, y), \frac{d(x, T x)+d(y, T y)}{2}\right\} .
$$

\section{Fixed Point Theorem on Rectangular Metric Spaces}

We introduce a new notion of generalized $\theta-\phi$-expansive mapping in the context of rectangular metric spaces as follows.

Definition 13. Let $(X, d)$ be a rectangular metric space and $T: X \longrightarrow X$ be a given mapping. $T$ is said to be generalized $\theta-\phi$-expansive mapping if there exists two functions $\theta \epsilon$ $\Theta$ and $\phi \in \Phi$ such that

$M(x, y)>0 \Longrightarrow \phi[\theta(d(T x, T y))] \geq \theta(M(x, y))$, for all $x, y \in X$,

where

$$
M(x, y)=\min \{d(x, y), d(x, T x), d(y, T y), d(x, T y)\} .
$$

Theorem 14. Let $(X, d)$ be a $(\alpha, \eta)$-complete generalized metric space, and $T: X \longrightarrow X$ be a bijective generalized $\theta-$ $\phi$-expansive mapping satisfying the following conditions:

(i) $T^{-1}$ is a triangular $(\alpha, \eta)$-admissible mapping

(ii) There exists $x_{0} \in X$ such that $\alpha\left(x_{0}, T^{-1} x_{0}\right) \geq 1$ or $\eta$ $\left(x_{0}, T^{-1} x_{0}\right) \leq 1$

(iii) Tis a $(\alpha, \eta)$ - continuous

Then, $T$ has a fixed point. Moreover, $T$ has a unique fixed point when $\alpha(z, u) \geq 1$ or $\eta(z, u) \leq 1$ for all $z, u \in \operatorname{Fix}(T)$.

Proof. Let $x_{0} \in X$ such that $\alpha\left(x_{0}, T^{-1} x_{0}\right) \geq 1$ or $\eta\left(x_{0}, T^{-1} x_{0}\right)$ $\leq 1$. We define the sequence $\left\{x_{n}\right\}$ in $X$ by $x_{n}=T x_{n+1}$, for all $n \in \mathbb{N}$.
Since $T^{-1}$ is a triangular $(\alpha, \eta)$-admissible mapping, then

$\alpha\left(x_{0}, x_{1}\right)=\alpha\left(x_{0}, T^{-1} x_{0}\right) \geq 1 \Longrightarrow \alpha\left(T^{-1} x_{0}, T^{-1} x_{1}\right)=\alpha\left(x_{1}, x_{2}\right) \geq 1$

or

$$
\eta\left(x_{0}, x_{1}\right)=\eta\left(x_{0}, T^{-1} x_{0}\right) \leq 1 \Longrightarrow \eta\left(T^{-1} x_{0}, T^{-1} x_{1}\right)=\eta\left(x_{1}, x_{2}\right) \leq 1 .
$$

Continuing this process, we have

$$
\alpha\left(x_{n-1}, x_{n}\right) \geq 1
$$

or

$$
\eta\left(x_{n-1}, x_{n}\right) \leq 1,
$$

for all $n \in \mathbb{N}$. By $\left(\mathrm{T}_{3}\right)$ and $\left(\mathrm{T}_{4}\right)$, one has.

$$
\alpha\left(x_{m}, x_{n}\right) \geq \operatorname{lor} \eta\left(x_{m}, x_{n}\right) \leq 1, \forall m, n \in \mathbb{N}, m \neq n .
$$

Suppose that there exists $n_{0} \in \mathbb{N}$ such that $x_{n_{0}}=T x_{n_{0}}$. Then, $x_{n_{0}}$ is a fixed point of $T$ and the proof is finished. Hence, we assume that $x_{n} \neq T x_{n}$, i.e., $d\left(x_{n-1}, x_{n}\right)>0$ for all $n \in \mathbb{N}$.

Step 1: We shall prove

$$
\lim _{n \rightarrow+\infty} d\left(x_{n}, x_{n+1}\right)=0 .
$$

Applying inequality (11) with $x=x_{n}$ and $y=x_{n+1}$, we obtain

$$
\begin{aligned}
\theta\left(d\left(x_{n-1}, x_{n}\right)\right) & =\theta\left(d\left(T x_{n}, T x_{n+1}\right)\right) \\
& >\phi\left[\theta\left(d\left(T x_{n}, T x_{n+1}\right)\right)\right] \\
& \geq \theta\left(M\left(x_{n}, x_{n+1}\right)\right),
\end{aligned}
$$

where

$$
\begin{aligned}
M\left(x_{n}, x_{n+1}\right) & =\min \left\{d\left(x_{n}, x_{n+1}\right), d\left(x_{n}, T x_{n}\right), d\left(x_{n+1}, T x_{n+1}\right), d\left(x_{n}, T x_{n+1}\right)\right\} \\
& =\min \left\{d\left(x_{n}, x_{n+1}\right), d\left(x_{n}, x_{n-1}\right), d\left(x_{n+1}, x_{n}\right), d\left(x_{n}, x_{n}\right)\right\} \\
& =\min \left\{d\left(x_{n}, x_{n+1}\right), d\left(x_{n}, x_{n-1}\right)\right\} .
\end{aligned}
$$

If for some $n, M\left(x_{n}, x_{n+1}\right)=d\left(x_{n}, x_{n-1}\right)$, then the inequality (19), we get

$$
\theta\left(d\left(x_{n-1}, x_{n}\right)\right)>\phi\left[\theta\left(d\left(T x_{n}, T x_{n+1}\right)\right)\right] \geq \theta\left(d\left(x_{n-1}, x_{n}\right)\right),
$$

It is a contradiction. Hence, $M\left(x_{n}, x_{n+1}\right)=d\left(x_{n}, x_{n+1}\right)$. Therefore,

$$
\phi\left[\theta\left(d\left(T x_{n}, T x_{n+1}\right)\right)\right] \geq \theta\left(d\left(x_{n}, x_{n+1}\right)\right) .
$$


Thus,

$$
\theta\left(d\left(x_{n}, x_{n+1}\right)\right) \leq \phi\left[\theta\left(d\left(x_{n-1}, x_{n}\right)\right)\right] \text { for all } n \in \mathbb{N}
$$

Continuing this process, we get

$$
\theta\left(d\left(x_{n}, x_{n+1}\right)\right) \leq \phi\left[\theta\left(d\left(x_{n-1}, x_{n}\right)\right)\right] \leq \cdots \leq \phi^{n}\left[\theta\left(d\left(x_{0}, x_{1}\right)\right)\right] .
$$

Now, by (23) and (2.9), we deduce that

$$
d\left(x_{n}, x_{n+1}\right)<d\left(x_{n-1}, x_{n}\right) .
$$

By the property of $\theta$ and $\phi$, it is evident that

$$
\lim _{n \rightarrow+\infty} d\left(x_{n}, x_{n+1}\right)=0
$$

Step 2. Now, we shall prove

$$
x_{n} \neq x_{m} \text {, for all } m, n \in \mathbb{N}, m \neq n .
$$

On the contrary, assume that $x_{n}=x_{m}$ for some $n=m+$ $k>m$. Indeed, suppose that $x_{n}=x_{m}$, so we have

$$
x_{n}=T x_{n+1}=T x_{m+1}=x_{m} .
$$

Denote $d_{n}=d\left(x_{n}, x_{n+1}\right)$. By inequality (25), we have

$$
d_{n}<d_{n-1} .
$$

Continuing this process, we get

$$
d_{m}=d_{n}<d_{n-1}<\cdots<d_{m}
$$

which is a contradiction. Thus, (27) hold.

Step 3. We shall prove

$$
\lim _{n \rightarrow+\infty} d\left(x_{n}, x_{n+2}\right)=0
$$

Applying inequality (11) with $x=x_{n}, y=x_{n+2}$, we obtain

$$
\begin{aligned}
\theta\left(d\left(x_{n-1}, x_{n+1}\right)\right) & =\theta\left(d\left(T x_{n}, T x_{n+2}\right)\right) \\
& >\phi\left[\theta\left(d\left(T x_{n}, T x_{n+2}\right)\right)\right] \\
& \geq \theta\left(M\left(x_{n}, x_{n+2}\right)\right)
\end{aligned}
$$

where

$$
\begin{aligned}
M\left(x_{n}, x_{n+2}\right) & =\min \left\{d\left(x_{n}, x_{n+2}\right), d\left(x_{n}, T x_{n}\right), d\left(x_{n+2}, T x_{n+2}\right), d\left(x_{n}, T x_{n+2}\right)\right\} \\
& =\min \left\{d\left(x_{n}, x_{n+2}\right), d\left(x_{n}, x_{n-1}\right), d\left(x_{n+2}, x_{n+1}\right), d\left(x_{n}, x_{n+1}\right)\right\} \\
& =\min \left\{d\left(x_{n}, x_{n+2}\right), d\left(x_{n+1}, x_{n+2}\right)\right\} .
\end{aligned}
$$

Take $a_{n}=d\left(x_{n}, x_{n+2}\right)$ and $b_{n}=d\left(x_{n+1}, x_{n+2}\right)$. Thus, by
(32), one can write

$$
\begin{aligned}
\theta\left(a_{n-1}\right) & =\theta\left(d\left(x_{n-1}, x_{n+1}\right)\right) \\
& =\theta\left(d\left(T x_{n}, T x_{n+2}\right)\right) \\
& >\phi\left[\theta\left(d\left(T x_{n}, T x_{n+2}\right)\right)\right] \\
& \geq \theta\left[M\left(x_{n}, x_{n+2}\right)\right] \\
& =\theta\left(\min \left\{a_{n}, b_{n}\right\}\right) .
\end{aligned}
$$

Therefore,

$$
a_{n-1} \geq \min \left\{a_{n}, b_{n}\right\} \text {. }
$$

Again, by (25),

$$
b_{n-1} \geq b_{n} \geq \min \left\{a_{n}, b_{n}\right\}
$$

which implies that

$$
\min \left\{a_{n}, b_{n}\right\} \leq \min \left\{a_{n-1}, b_{n-1}\right\}, \forall n \in \mathbb{N} .
$$

Then, the sequence $\min \left\{a_{n}, b_{n}\right\}_{n \in \mathbb{N}}$ is monotone nonincreasing. Thus, there exists $\lambda \geq 0$ such that

$$
\lim _{n \rightarrow \infty} \min \left\{a_{n}, b_{n}\right\}=\lambda
$$

Assume that $\lambda>0$. By (26), we have

$\lim _{n \rightarrow \infty} \sup a_{n}=\lim _{n \rightarrow \infty} \operatorname{supmin}\left\{a_{n}, b_{n}\right\}=\lim _{n \rightarrow \infty} \min \left\{a_{n}, b_{n}\right\}=\lambda$.

Taking the limsup $n \rightarrow \infty$ in (32), and using $\left(\theta_{3}\right),\left(\phi_{3}\right)$, and Lemma 9, we obtain

$$
\begin{aligned}
\theta(\lambda) & =\theta\left(\lim _{n \rightarrow \infty} \sup a_{n-1}\right) \\
& \geq \phi\left[\theta\left(\lim _{n \rightarrow \infty} \sup \left\{a_{n}\right\}\right)\right] \\
& \geq \theta\left(\lim _{n \rightarrow \infty} \min \left\{a_{n}, b_{n}\right\}\right),
\end{aligned}
$$

which implies that

$$
\theta(\lambda) \geq \phi[\theta(\lambda)] \geq \theta(\lambda)
$$

Therefore,

$$
\theta(\lambda)<\theta(\lambda)
$$

By $\left(\theta_{1}\right)$, we get

$$
\lambda<\lambda
$$

It is a contradiction, then

$$
\lim _{n \rightarrow \infty} d\left(x_{n}, x_{n+2}\right)=0 .
$$


Step 4 . We shall prove that $\left\{x_{n}\right\}$ is a Cauchy sequence in $(X, d)$, that is,

$$
\lim _{n, m \rightarrow \infty} d\left(x_{n}, x_{m}\right)=0 \text { for all } n, m \in \mathbb{N}, m>n
$$

If otherwise there exists an $\varepsilon>0$ for which we can find sequence of positive integers $\left\{x_{n_{(k)}}\right\}_{k}$ and $\left\{x_{m_{(k)}}\right\}_{k}$ of $\left\{x_{n}\right\}$ such that, for all positive integers $k, n_{(k)}>m_{(k)}>k$,

$$
\begin{gathered}
d\left(x_{m_{(k)}}, x_{n_{(k)}}\right) \geq \varepsilon, \\
d\left(x_{m_{(k)}}, x_{n_{(k)-1}}\right)<\varepsilon .
\end{gathered}
$$

Now, using (26), (44), (46), and the rectangular inequality, we find

$$
\begin{aligned}
\varepsilon \leq & d\left(x_{m_{(k)}}, x_{n_{(k)}}\right) \leq d\left(x_{m_{(k)}}, x_{m_{(k)+1}}\right) \\
& +d\left(x_{m_{(k)+1}}, x_{m_{(k)-1}}\right)+d\left(x_{m_{(k)-1}}, x_{n_{(k)}}\right) \\
< & d\left(x_{m_{(k)}}, x_{m_{(k)+1}}\right)+d\left(x_{m_{(k)+1}}, x_{m_{(k)}-1}\right)+\varepsilon .
\end{aligned}
$$

Then,

$$
\lim _{k \rightarrow \infty} d\left(x_{m_{(k)}}, x_{n_{(k)}}\right)=\varepsilon
$$

Now, by rectangular inequality, we have

$$
\begin{gathered}
d\left(x_{m_{(k)+1}}, x_{n_{(k)+1}}\right) \leq d\left(x_{m_{(k)+1}}, x_{m_{(k)}}\right)+d\left(x_{m_{(k)}}, x_{n_{(k)}}\right)+d\left(x_{n_{(k)}}, x_{n_{(k)+1}}\right), \\
d\left(x_{m_{(k)}}, x_{n_{(k)}}\right) \leq d\left(x_{m_{(k)}}, x_{m(k)+1}\right)+d\left(x_{m_{(k)+1}}, x_{n_{(k)+1}}\right)+d\left(x_{n_{(k)+1}}, x_{n_{(k)}}\right), \\
\varepsilon \leq d\left(x_{m_{(k)}}, x_{n_{(k)}}\right) \leq d\left(x_{m_{(k)}}, x_{n_{(k)-1}}\right)+d\left(x_{n_{(k)-1}}, x_{n_{(k)+1}}\right)+d\left(x_{n_{(k)+1}}, x_{n_{(k)}}\right) .
\end{gathered}
$$

Letting $k \longrightarrow \infty$ in the above inequalities, using (26), (46), and (49), we obtain

$$
\begin{gathered}
\lim _{k \rightarrow \infty} d\left(x_{m_{(k)+1}}, x_{n_{(k)+1}}\right)=\varepsilon, \\
\lim _{k \rightarrow \infty} d\left(x_{m_{(k)}}, x_{n_{(k)-1}}\right)=\varepsilon .
\end{gathered}
$$

On the other hand,

$$
\begin{aligned}
M\left(x_{m_{(k)}}, x_{n_{(k)}}\right)= & \min \left\{d\left(x_{m_{(k)}}, x_{n_{(k)}}\right), d\left(x_{m_{(k)}}, T x_{m_{(k)}}\right), d\right. \\
& \left.\cdot\left(x_{n_{(k)}}, T x_{n_{(k)}}\right), d\left(x_{m_{(k)}}, T x_{n_{(k)}}\right)\right\} \\
= & \min \left\{d\left(x_{m_{(k)}}, x_{n_{(k)}}\right), d\left(x_{m_{(k)}}, x_{m_{(k)-1}}\right), d\right. \\
& \left.\cdot\left(x_{n_{(k)}}, x_{n_{(k)-1}}\right), d\left(x_{m_{(k)}}, x_{n_{(k)-1}}\right)\right\} .
\end{aligned}
$$

Letting $k \longrightarrow \infty$ in the above inequalities and using (26), (49), and (52), we get that

$$
\lim _{k \rightarrow \infty} M\left(x_{m_{(k)}}, x_{n_{(k)}}\right)=\varepsilon
$$

By (51), let $B=\varepsilon / 2>0$, from the definition of the limit, there exists $n_{0} \in \mathbb{N}$ such that

$$
\left|d\left(x_{m_{(k)+1}}, x_{n_{(k)+1}}\right)-\varepsilon\right| \leq B \forall n \geq n_{0} .
$$

This implies that

$$
d\left(x_{m_{(k)+1}}, x_{n_{(k)+1}}\right) \geq B>0 \forall n \geq n_{0},
$$

and by (54), let $A=\varepsilon / 2>0$, from the definition of the limit, there exists $n_{1} \in \mathbb{N}$ such that

$$
M\left(x_{m_{(k)}}, x_{n_{(k)}}\right) \geq A>0 \forall n \geq n_{1} .
$$

Applying (11) with $x=x_{m_{(k)}}$ and $y=x_{n_{(k)}}$, we obtain

$$
\phi\left[\theta\left(d\left(x_{m_{(k)+1}}, x_{n_{(k)+1}}\right)\right] \geq \theta\left(M\left(d\left(x_{m_{(k)}}, x_{n_{(k)}}\right)\right) .\right.\right.
$$

Letting $k \longrightarrow \infty$, the above inequality and using $\left(\theta_{3}\right)$ and $\left(\phi_{3}\right)$, we obtain

$$
\phi\left[\theta\left(\lim _{k \rightarrow \infty} d\left(x_{m_{(k)+1}}, x_{n_{(k)+1}}\right)\right)\right] \geq \theta\left(\lim _{k \rightarrow \infty} M\left(x_{m_{(k)}}, x_{n_{(k)}}\right)\right) .
$$

Therefore,

$$
\phi(\theta(\varepsilon)) \geq \theta(\varepsilon)
$$

It is a contradiction with Lemma 9, according to which

$$
\phi(\theta(\varepsilon))<\theta(\varepsilon)
$$

Then,

$$
\lim _{n, m \rightarrow \infty} d\left(x_{m}, x_{n}\right)=0
$$

It follows that $\left\{x_{n}\right\}$ is a Cauchy sequence in $X$. Since $(X, d)$ is $(\alpha, \eta)$-complete and

$$
\begin{gathered}
\alpha\left(x_{n-1}, x_{n}\right) \geq 1 \\
\text { or } \eta\left(x_{n-1}, x_{n}\right) \leq 1,
\end{gathered}
$$

for all $n \in \mathbb{N}$, the there exists $z \in X$ such that

$$
\lim _{x \rightarrow \infty} d\left(x_{n}, z\right)=0 .
$$


Step 5 . We show that $d(T z, z)=0$ arguing by contradiction, we assume that

$$
d(T z, z)>0
$$

By rectangular inequality, we get

$$
\begin{gathered}
d\left(T x_{n}, T z\right) \leq d\left(T x_{n}, x_{n}\right)+d\left(x_{n}, z\right)+d(z, T z), \\
d(z, T z) \leq d\left(z, x_{n}\right)+d\left(x_{n}, T x_{n}\right)+d\left(T x_{n}, T z\right) .
\end{gathered}
$$

By letting $n \longrightarrow \infty$ in inequality (66) and (67), we obtain

$$
d(z, T z) \leq \lim _{n \rightarrow \infty} d\left(T x_{n}, T z\right) \leq d(z, T z) .
$$

Therefore,

$$
\lim _{n \rightarrow \infty} d\left(T x_{n}, T z\right)=d(z, T z)
$$

Since $T$ is $(\alpha, \eta)$-continuous, then $T x_{n} \longrightarrow T z$ i.e $\lim _{n \rightarrow \infty} d\left(T x_{n}, T z\right)=0$. Hence, $d(T z, z)=0$, so $T z=z$.

Step 6. Uniqueness. Now, suppose that $z, u \in X$ are two fixed points of $T$ such that $u \neq z$ and $\alpha(z, u) \geq 1$ or $\eta(z, u)$ $\leq 1$. Therefore, we have

$$
d(T z, T u)=d(z, u)>0
$$

Applying (11) with $x=z$ and $y=u$, we have

$$
\phi[\theta(d(T u, T z))] \geq[\theta(M(z, u))],
$$

where

$M(z, u)=\min \{d(z, u), d(z, T z), d(u, T u), d(z, T u)\}=d(z, u)$.

Therefore, we have

$$
\theta(d(z, u))>\phi[\theta(d(u, z))] \geq[\theta(d(z, u))]
$$

It is a contradiction. Therefore, $u=z$.

Theorem 15. Let $\alpha, \eta: X \times X \longrightarrow \mathbb{R}^{+}$be two functions and let $(X, d)$ be a $(\alpha, \eta)$ - complete rectangular metric space. Let $T: X \longrightarrow X$ be a bijective, mapping satisfying the following conditions:

(i) $T^{-1}$ is a triangular $(\alpha, \eta)$-admissible mapping

(ii) $T$ is a generalized $(\alpha, \eta)-\theta-\phi$-expansive mapping

(iii) $\alpha\left(z, T^{-1} z\right) \geq 1$ or $\eta\left(z, T^{-1} z\right) \leq 1$, for all $z \in$ Fix $(T)$

Then, $T$ has the property $P$.
Proof. Let $z \in \operatorname{Fix}\left(T^{n}\right)$ for some fixed $n>1$. As $\alpha\left(z, T^{-1} z\right) \geq 1$ or $\eta\left(z, T^{-1} z\right) \leq 1$ and $T^{-1}$ is a triangular $(\alpha, \eta)$-admissible mapping, then

$$
\begin{gathered}
\alpha\left(T^{-1} z, T^{-2} z\right) \geq 1 \\
\text { or } \eta\left(T^{-2} z, T^{-1} z\right) \leq 1 .
\end{gathered}
$$

Continuing this process, we have

$$
\begin{aligned}
\alpha\left(T^{-n} z, T^{-n-1} z\right) & \geq 1 \\
\text { or } \eta\left(T^{-n} z, T^{-n-1} z\right) & \leq 1,
\end{aligned}
$$

for all $n \in \mathbb{N}$. By (T3) and (T4), we get

$$
\begin{gathered}
\alpha\left(T^{-m} z, T^{-n} z\right) \geq 1 \\
\text { or } \eta\left(T^{-m} z, T^{-n} z\right) \leq 1, \forall m, n \in \mathbb{N}, n \neq m .
\end{gathered}
$$

Since $T$ is a bijective mapping, then $T^{-n} z=z=T^{n} z$ for all $n \in \mathbb{N}$ and $z \in \operatorname{Fix}(T)$. Therefore,

$$
\begin{gathered}
\alpha\left(T^{m} z, T^{n} z\right) \geq 1 \\
\text { or } \eta\left(T^{m} z, T^{n} z\right) \leq 1, \forall m, n \in \mathbb{N}, n \neq m .
\end{gathered}
$$

Assume that $z \notin F i x(T)$, i.e., $d(z, T z)>0$. Then, we have

$$
d(z, T z)=d\left(T^{n} z, T z\right)=d\left(T T^{n-1} z, T z\right) .
$$

Applying (11) with $x=z$ and $y=T^{n-1} z$, we obtain

$$
\phi[\theta(d(z, T z))]=\phi\left[\theta\left(d\left(T T^{n-1} z, T z\right)\right)\right] \geq \theta\left(M\left(T^{n-1} z, z\right)\right),
$$

where

$$
\begin{aligned}
M\left(T^{n-1} z, z\right)= & \min \left\{d\left(z, T^{n-1} z\right), d(z, T z), d\right. \\
& \left.\cdot\left(T^{n-1} z, T T^{n-1} z\right), d\left(T^{n-1} z, T z\right)\right\}
\end{aligned}
$$

Letting $n \longrightarrow \infty$ in (80), we obtain

$$
\lim _{n \rightarrow+\infty} M\left(T^{n-1} z, z\right)=d(z, T z)
$$

Now, using $\left(\theta_{3}\right)$ and $\left(\phi_{3}\right)$, we get

$$
\phi[\theta(d(z, T z))] \geq \theta(d(z, T z)) .
$$

It is a contradiction. Then, $z \in \operatorname{Fix}(T)$.

Example 16. Let $X=[1,+\infty[$ and $d: X \times X \longrightarrow[0,+\infty[$ defined by

$$
d(x, y)=|x-y|
$$

Then, $(X, d)$ is a metric space and rectangular metric 
space. Define mapping $T: X \longrightarrow X$ and $\alpha, \eta: X \times X \longrightarrow$ $[0,+\infty[$ by

$$
\begin{aligned}
T(x) & =x^{4}, \\
\alpha(x, y) & =\frac{x+y}{\max \{x, y\}}, \\
\eta(x, y) & =\frac{|x-y|}{\max \{x, y\}} .
\end{aligned}
$$

Then, $T$ is an $(\alpha, \eta)$ - continuous triangular $(\alpha, \eta)-$ admissible mapping and $T$ is a bijective mapping.

Let $\theta(t)=\sqrt{t}+1, \phi(t)=(t+1) / 2$.

Evidently, $((\alpha(x, y) \geq 1$ or $(x, y) \leq 1)$ and $\min \{d(x, y), d$ $(x, T x), d(y, T y), d(y, T y)\}>0$ are when $x \neq y \neq 1$.

Consider two cases:

Case 1. $x>y>1$.

$$
\begin{aligned}
d(T x, T y) & =x^{4}-y^{4}, \theta(d(T x, T y)) \\
& =\sqrt{x^{4}-y^{4}}+1 \\
& =\sqrt{\left(x^{2}+y^{2}\right)(x+y)(x-y)}+1 .
\end{aligned}
$$

Thus,

$$
\phi[\theta(d(T x, T y))]=\sqrt{\frac{\left.x^{2}+y^{2}\right)(x+y)(x-y)}{4}}+1 .
$$

We have

$$
\theta(d(x, y)=x-y .
$$

On the other hand,

$$
\begin{aligned}
\theta(d(x, y))-\phi[\theta(d(T x, T y))] & =x-y+1-\sqrt{\frac{\left.x^{2}+y^{2}\right)(x+y)(x-y)}{4}}-1 \\
& =x-y-\sqrt{\frac{\left.x^{2}+y^{2}\right)(x+y)(x-y)}{4}}
\end{aligned}
$$

Since $x, y \in] 1,+\infty[$, then

$$
x-y-\sqrt{\frac{\left.x^{2}+y^{2}\right)(x+y)(x-y)}{4}} \leq 0,
$$

which implies that

$$
\begin{aligned}
\phi[\theta(d(T x, T y))] & \geq \theta(d(x, y)) \\
& \geq \theta[\min \{d(x, y), d(x, T x), d(y, T y), d(y, T y)] .
\end{aligned}
$$

Case 2. $y>x>1$.

$$
\begin{aligned}
d(T x, T y) & =y^{4}-x^{4}, \theta(d(T x, T y)) \\
& =\sqrt{y^{4}-x^{4}}+1 \\
& =\sqrt{\left(y^{2}+x^{2}\right)(y+x)(y-x)}+1 .
\end{aligned}
$$

Thus,

$$
\phi[\theta(d(T x, T y))]=\sqrt{\frac{\left.x^{2}+y^{2}\right)(x+y)(y-x)}{4}}+1
$$

We have

$$
\theta(d(x, y))=y-x .
$$

On the other hand

$$
\begin{aligned}
\theta(d(x, y))-\phi[\theta(d(T x, T y))] & =x-y+1-\sqrt{\frac{\left.x^{2}+y^{2}\right)(x+y)(y-x)}{4}}-1 \\
& =y-x-\sqrt{\frac{\left.x^{2}+y^{2}\right)(x+y)(y-x)}{4}} .
\end{aligned}
$$

Since $x, y \in] 1,+\infty[$, then

$$
y-x-\sqrt{\frac{\left.x^{2}+y^{2}\right)(x+y)(y-x)}{4}} \leq 0,
$$

which implies that

$$
\begin{aligned}
\phi[\theta(d(T x, T y))] & \geq \theta(d(x, y)) \\
& \geq \theta[\min \{d(x, y), d(x, T x), d(y, T y), d(y, T y)]
\end{aligned}
$$

Hence, condition (11) is satisfied. Therefore, $T$ has a unique fixed point $z=1$.

Theorem 17. Let $\alpha, \eta: X \times X \longrightarrow \mathbb{R}^{+}$be two functions and let $d(X, d)$ be a $(\alpha, \eta)$ - complete rectangular metric space. Let $T: X \longrightarrow X$ be a bijective mapping satisfying the following assertions:

(i) $T^{-1}$ is triangular $(\alpha, \eta)$-admissible

(ii) $T$ is a generalized $(\alpha, \eta)-\theta-\phi$-expansive mapping

(iii) There exists $x_{0} \in X$ such that $\alpha\left(x_{0}, T^{-1} x_{0}\right) \geq 1$ or $\eta$ $\left(x_{0}, T^{-1} x_{0}\right) \leq 1$

(iv) $(X, d)$ is a $(\alpha, \eta)$-regular rectangular metric space 
Then, $T$ has a fixed point. Moreover, $T$ has a unique fixed point whenever $\alpha(z, u) \geq 1$ or $\eta(z, u) \leq 1$ for all $z, u \in \operatorname{Fix}(T)$.

Proof. Let $\mathrm{x}_{0} \in X$ such that $\alpha\left(x_{0}, T^{-1} x_{0}\right) \geq 1$ or $\eta\left(x_{0}, T^{-1} x_{0}\right)$ $\leq 1$. Similar to the proof of Theorem 14 , we can conclude that

$$
\left(\alpha\left(x_{n}, x_{n+1}\right) \geq 1 \text { or } \eta\left(x_{n}, x_{n+1}\right) \leq 1\right), x_{n} \longrightarrow z \text {, as } n \longrightarrow \infty \text {, }
$$

and from inequality (69), we have

$$
\lim _{n \rightarrow \infty} d\left(T x_{n}, T z\right)=d(z, T z) .
$$

From (iv) $\alpha\left(x_{n}, z\right) \geq 1$ or $\eta\left(x_{n}, z\right) \leq 1$ hold for $n \in \mathbb{N}$.

Suppose that $T z=x_{n_{0-1}}=T x_{n_{0}}$ for some $n_{0} \in \mathbb{N}^{*}$. From Theorem 14, we know that the members of the sequence $\left\{x_{n}\right\}$ are distinct. Hence, we have $T z \neq T x_{n}$, i.e., $d(T z, T$ $\left.x_{n}\right)>0$ for all $n>n_{0}$. Thus, we can apply (11), to $x_{n}$ and $z$ for all $n>n_{0}$ to get

$$
\phi\left[\theta\left(d\left(T z, T x_{n}\right)\right)\right] \geq\left[\theta\left(M\left(z, x_{n}\right)\right)\right], \forall n \geq n_{0},
$$

where

$$
\begin{aligned}
M\left(z, x_{n}\right) & =\min \left\{d\left(z, x_{n}\right), d(z, T z), d\left(x_{n}, T x_{n}\right), d\left(z, T x_{n}\right)\right\} \\
& =\min \left\{d\left(z, x_{n}\right), d(z, T z), d\left(x_{n}, x_{n-1}\right), d\left(z, x_{n-1}\right)\right\}
\end{aligned}
$$

Therefore,

$$
\begin{aligned}
\phi\left[\theta\left(d\left(T z, T x_{n}\right)\right)\right] \geq & {\left[\theta \left(\operatorname { m i n } \left\{d\left(z, x_{n}\right), d(z, T z), d\right.\right.\right.} \\
& \left.\left.\left.\cdot\left(x_{n}, x_{n-1}\right), d\left(z, x_{n-1}\right)\right\}\right)\right] .
\end{aligned}
$$

By letting $n \longrightarrow \infty$ in inequality (101), we obtain

$$
\begin{aligned}
\lim _{n \rightarrow \infty} \phi\left[\theta\left(d\left(T z, T x_{n}\right)\right)\right] \geq & \lim _{n \rightarrow \infty}\left[\theta \left(\operatorname { m i n } \left\{d\left(z, x_{n}\right), d\right.\right.\right. \\
& \left.\left.\left.\cdot(z, T z), d\left(x_{n}, x_{n-1}\right), d\left(z, x_{n-1}\right)\right\}\right)\right] .
\end{aligned}
$$

Since $\theta$ and $\phi$ are continuous functions and $\lim _{n \rightarrow+\infty} M$ $\left(z, x_{n}\right)=d(z, T z)$, we conclude that

$$
\phi[\theta(d(z, T z))] \geq[\theta(d(z, T z))] .
$$

which implies that

$$
d(z, T z)<d(z, T z)
$$

It is a contradiction. Hence, $T z=z$.

The proof of the uniqueness is similarly to that of Theorem 14.

Corollary 18. Let $\alpha, \eta: X \times X \longrightarrow[0,+\infty[$ be two functions, $(X, d)$ be a $(\alpha, \eta)$-complete rectangular metric space and
$T: X \longrightarrow X$ be a bijective mapping. Suppose that for all $x, y$ $\in X$ with $\alpha(x, y) \geq 1$ or $\eta(x, y) \leq 1$ and $M(x, y)>0$ we have

$$
[\theta(d(T x, T y))]^{k} \geq \theta(M(x, y))
$$

where

$$
M(x, y)=\min \{d(x, y), d(x, T z), d(y, T y), d(x, T y)\}
$$

and $k \in] 0,1[, \theta \in \Theta$. Then, $T$ has a fixed point, if

(i) $T^{-1}$ is a triangular $(\alpha, \eta)$-admissible mapping

(ii) there exists $x_{0} \in X$ such that $\alpha\left(x_{0}, T^{-1} x_{0}\right) \geq 1$ or $\eta$ $\left(x_{0}, T^{-1} x_{0}\right) \leq 1$

(iii) Tis a $(\alpha, \eta)$ - continuous or

(iv) $(X, d)$ is an $(\alpha, \eta)$-regular rectangular metric space

Moreover, $T$ has a unique fixed point when $\alpha(z, u) \geq 1$ or $\eta(z, u) \leq 1$ for all $z, u \in \operatorname{Fix}(T)$.

\section{Fixed Point Theorem on Rectangular Metric Spaces Endowed with a Partial Order}

Definition 19 (see [4]). Let $(X, d, \preceq)$ be an ordered rectangular metric space and $T: X \longrightarrow X$ be a mapping.

(1) $(X, d)$ is said to be $O$-complete, if every Cauchy $\left\{x_{n}\right\}$ in $X$ with $x_{n} \preceq x_{n+1}$ for all $n \in \mathbb{N}$ or $x_{n} \pm x_{n+1}$ for all $n$ $\in \mathbb{N}$ converges in $X$

(2) $(X, d)$ is said to be $O$-regular, if for each sequence $\left\{x_{n}\right\}$ in $X, x_{n} \longrightarrow x$ and $x_{n} \geq x_{n+1}$ for all $n \in \mathbb{N}$ or $x_{n} \pm x_{n+1}$ for all $n \in \mathbb{N}$ imply that $x_{n} \preceq x$ or $x_{n} \pm x$, respectively

(3) $T$ is said to be $O$-continuous, if for given $x \in X$ and sequence $\left\{x_{n}\right\}$ with $x_{n} \geq x_{n+1}$ or $x_{n} \preceq x_{n+1}$ for all $n \in$ $\mathbb{N}, x_{n} \longrightarrow x \Rightarrow T x_{n} \longrightarrow T x$

Definition 20. Let $(X, d, \preceq)$ be an ordered rectangular metric spaces and $T: X \longrightarrow X$ be a mapping. We say that $T$ aids to be an ordered $\theta-\phi$-expansive mapping, if for all $x, y \in X$ with $x \preceq y$ or $x \geq y$ such that

$$
M(x, y)>0 \Longrightarrow \phi[\theta(d(T x, T y))] \geq \theta(M(x, y))
$$

where

$$
M(x, y)=\min \{d(x, y), d(x, T x), d(y, T y), d(x, T y)\}
$$

Theorem 21. Let $(X, d, \preceq)$ be an $O$-complete partially ordered rectangular metric space. Let $T: X \longrightarrow X$ be a bijective self mapping on $X$ satisfying the following assertions: 
(i) $T^{-1}$ is monotone

(ii) $T$ is an ordered $\theta-\phi$-expansive mapping

(iii) there exists $x_{0} \in X$ such that $x_{0} \preceq T^{-1} x_{0}$ or $x_{0} \geq T^{-1} x_{0}$

(iv) either $T$ is O-continuous or

(v) $(X, d)$ is O-regular

Then, Thas a fixed point. Moreover, T has a unique fixed point whenever $z \preceq u$ or $z \succeq u$ for all $z, u \in$ Fix $(T)$.

Proof. Define the mapping $\alpha: X \times X \longrightarrow[0,+\infty[$ by

$$
\alpha(x, y)= \begin{cases}1, & \text { if } x \leq y \\ 0, & \text { otherwise }\end{cases}
$$

and the mapping $\eta: X \times X \longrightarrow[0,+\infty[$ by

$$
\eta(x, y)= \begin{cases}1, & \text { if } x \geq y \\ 0, & \text { otherwise }\end{cases}
$$

Using condition (iii), we have

$$
\begin{aligned}
x_{0} \leq T^{-1} x_{0} & \Longrightarrow \alpha\left(x_{0}, T^{-1} x_{0}\right) \geq 1 \\
\text { or } x_{0} \geq T^{-1} x_{0} & \Longrightarrow \eta\left(x_{0}, T^{-1} x_{0}\right) \leq 1 .
\end{aligned}
$$

Owing to the monotonicity of $T^{-1}$, we get

$$
\begin{gathered}
\alpha(x, y) \geq 1 \Longrightarrow x \leq y \Longrightarrow T^{-1} x^{\circ} T^{-1} y \Longrightarrow \alpha\left(T^{-1} x, T^{-1} y\right) \geq 1 \\
\text { or } \eta(x, y) \leq 1 \Longrightarrow x \geq y \Longrightarrow T^{-1} x \pm T^{-1} y \Longrightarrow \eta\left(T^{-1} x, T^{-1} y\right) \leq 1
\end{gathered}
$$

Therefore, $\left(\mathrm{T}_{1}\right)$ and $\left(\mathrm{T}_{2}\right)$ hold.

On the other hand, if

$$
\left\{\begin{array} { l } 
{ \alpha ( x , y ) \geq 1 } \\
{ \alpha ( x , y ) \geq 1 }
\end{array} \Longrightarrow \left\{\begin{array}{l}
x \leq y, \\
y \preceq z
\end{array}\right.\right.
$$

or

$$
\left\{\begin{array} { l } 
{ \eta ( x , y ) \leq 1 } \\
{ \eta ( x , y ) \leq 1 }
\end{array} \Longrightarrow \left\{\begin{array}{l}
x \geq y, \\
y \geq z .
\end{array}\right.\right.
$$

Since $(X, d)$ be an $O$-complete partially ordered rectangular metric space, we conclude that

$$
x \leq z \text { or } x \geq z \Longrightarrow \alpha(x, z) \geq 1 \text { or } \eta(x, z) \leq 1
$$

Thus, $\left(\mathrm{T}_{3}\right)$ and $\left(\mathrm{T}_{4}\right)$ hold. This shows that $T^{-1}$ is a triangular $(\alpha, \eta)$-admissible mapping then

$$
\left(\alpha\left(x_{n}, x_{n+1}\right) \geq 1 \text { or } \eta\left(x_{n}, x_{n+1}\right) \leq 1\right) .
$$

Now, if $T$ is $O$-continuous, then $x_{n} \preceq x_{n+1}$ or $x_{n} \geq x_{n+1} \Longrightarrow \alpha\left(x_{n}, x_{n+1}\right) \geq 1$ or $\eta\left(x_{n}, x_{n+1}\right) \leq 1$ and $x_{n} \longrightarrow z$ as $n \longrightarrow \infty$ with $z \in X \Longrightarrow T x_{n} \longrightarrow T x$.

The existence and uniqueness of a fixed point follows from Theorem 14.

Now, suppose that follow $(X, d, \preceq)$ is $O$-regular. Let $\left\{x_{n}\right\}$ be a sequence such that

$$
\left\{n_{n}\right\}^{\circ} x \text { or }\left\{n_{n}\right\} \geq x
$$

which implies that

$$
\left(\alpha\left(x_{n}, x\right) \geq 1 \text { or } \eta\left(x_{n}, x\right) \leq 1\right)
$$

for all $n$ and $x_{n} \longrightarrow x$ as $n \longrightarrow \infty$. This shows that $(X, d)$ is $(\alpha, \eta)$ - regular. Thus, the existence and uniqueness of fixed point from Theorem 17.

Corollary 22. Let $(X, d, \preceq)$ be an O-complete partially ordered rectangular metric spaces.

Let $T: X \longrightarrow X$ be a bijective self mapping on $X$ be such that $T^{-1}$ is a monotone mapping. Suppose that there exists $k \in] 0,1[$ such that

$$
k d(T x, T y) \geq d(x, y)
$$

for all $x, y \in X$ with $x \preceq y$ or $x \geq y$. Suppose also the following conditions hold:

(i) There exists $x_{0} \in X$ such that $x_{0} \preceq T^{-1} x_{0}$ or $x_{0} \succeq T^{-1} x_{0}$

(ii) Either $T$ is O-continuous or

(iii) $X$ is O-regular

Then, Thas a fixed point. Moreover, Thas a unique fixed point whenever $z \preceq u$ or $z \succeq u$ for all $z, u \in F i x(T)$.

Proof. By taking $\phi(t)=t^{\sqrt{k}}$ and $\theta(t)=e^{\sqrt{t}}$, obviously that $\phi \epsilon$ $\Phi$ and $\theta \in \Theta$ and $T$ is a $\theta-\phi$-expansive mapping. As in the proof of Theorem 21, we get the result.

\section{Conclusion}

In this article, we have proved some fixed point theorems for the $\theta-\phi$-expansive mapping in the settings of complete rectangular metric spaces. These results are extensions of the related results announced in $[6,7,9]$.

\section{Data Availability}

No data were used to support this study.

\section{Conflicts of Interest}

On behalf of all authors, the corresponding author states that there is no conflict of interest. 


\section{References}

[1] S. Banach, "Sur les opérations dans les ensembles abstraits et leur application aux équations intégrales," Fundamenta Mathematicae, vol. 3, pp. 133-181, 1922.

[2] A. Kari, M. Rossafi, E. M. Marhrani, and M. Aamri, "Fixed-Point Theorem for Nonlinear F-Contraction via $w$ -Distance," Advances in Mathematical Physics, vol. 2020, Article ID 6617517, 10 pages, 2020.

[3] A. Kari, M. Rossafi, H. Saffaj, E. Marhrani, and M. Aamri, "Fixed-Point Theorems for -Contraction in Generalized Asymmetric Metric Spaces," International Journal of Mathematics and Mathematical Sciences, vol. 2020, Article ID 8867020, 19 pages, 2020.

[4] A. Kari, M. Rossafi, E. M. Marhrani, and M. Aamri, "New Fixed Point Theorems for $\theta-\varphi$-Contraction on Rectangular -Metric Spaces," Abstract and Applied Analysis, vol. 2020, Article ID 8833214, 12 pages, 2020.

[5] A. Kari, M. Rossafi, E. Marhrani, and M. Aamri, "Contraction on Complete Rectangular Metric Spaces," International Journal of Mathematics and Mathematical Sciences, vol. 2020, Article ID 5689458, 9 pages, 2020.

[6] M. Kumar, S. M. Kang, P. Kumar, and S. Kumar, "Fixed point theorems for $\phi$-weakly expansive mappings in $G$-metric spaces," Pan American Mathematical Journal, vol. 24, no. 1, pp. 21-30, 2014.

[7] S. M. Kang, M. Kumar, P. Kumar, and S. Kumar, "Fixed point theorems for $\varphi$-WEAKLY expansive mappings in metric spaces," International Journal of Pure and Applied Mathematics, vol. 90, no. 2, pp. 143-152, 2014.

[8] S. Z. Wang, B. Y. Li, Z. M. Gao, and K. Iseki, "Some fixed point theorems on expansion mappings," Mathematica Japonica, vol. 29, pp. 631-636, 1984.

[9] M. Kumar, S. Araci, and P. Kumam, "Fixed point theorems for generalized $(\alpha, \psi)$ - expansive mappings in generalized metric spaces," Communications in Mathematics and Applications, vol. 7, no. 3, pp. 227-240, 2016.

[10] D. Zheng, Z. Cai, and P. Wang, "New fixed point theorems for $\theta-\varphi$ contraction in complete metric spaces," Journal of Nonlinear Sciences \& Applications, vol. 10, no. 5, pp. 2662-2670, 2017.

[11] A. Branciari, "A fixed point theorem of Banach-Caccioppoli type on a class of generalized metric spaces," Publicationes Mathematicae Debrecen, vol. 57, pp. 31-37, 2000.

[12] N. Hussain, M. A. Kutbi, and P. Salimi, "Fixed Point Theory in -Complete Metric Spaces with Applications," Abstract and Applied Analysis, vol. 2014, Article ID 280817, 11 pages, 2014.

[13] N. Hussain, V. Parvaneh, B. A. S. Alamri, and Z. Kadelburg, "F-HR-type contractions on $(\alpha-\eta)$-complete rectangular bmetric spaces," The Journal of Nonlinear Sciences and Applications, vol. 10, no. 3, pp. 1030-1043, 2017.

[14] M. Jleli, E. Karapınar, and B. Samet, "Further generalizations of the Banach contraction principle," Journal of Inequalities and Applications, vol. 2014, no. 1, 2014. 